\title{
Regulation of neuropathic pain behavior by amygdaloid TRPC4/C5 channels
}

\author{
Wei, Hong
}

2015-11-03

Wei , H , Sagalajev , B , Yuzer , M A , Koivisto , A \& Pertovaara , A 2015 , ' Regulation of neuropathic pain behavior by amygdaloid TRPC4/C5 channels ', Neuroscience Letters, vol. 608 , pp. 12-17 . https://doi.org/10.1016/j.neulet.2015.09.033

http://hdl.handle.net/10138/159280

https://doi.org/10.1016/j.neulet.2015.09.033

publishedVersion

Downloaded from Helda, University of Helsinki institutional repository.

This is an electronic reprint of the original article.

This reprint may differ from the original in pagination and typographic detail.

Please cite the original version. 
Research paper

\title{
Regulation of neuropathic pain behavior by amygdaloid TRPC4/C5 channels
}

\author{
Hong Wei ${ }^{\mathrm{a}, 1}$, Boriss Sagalajev ${ }^{\mathrm{a}, 1}$, M. Anil Yüzer ${ }^{\mathrm{a}}$, Ari Koivisto ${ }^{\mathrm{b}}$, Antti Pertovaara ${ }^{\mathrm{a}, *}$ \\ a Department of Physiology, Faculty of Medicine, University of Helsinki, Helsinki, Finland \\ b Orion Pharma, Orion Corporation, Turku, Finland
}

\section{H I G H L I G H T S}

- Blocking of amygdaloid TRPC4/C5 attenuated neuropathic hypersensitivity.

- Blocking of amygdaloid TRPC4/C5 attenuated affective-like neuropathic pain.

- In healthy rats, blocking amygdaloid TRPC4/C5 failed to influence pain behavior.

- Amygdaloid TRPC4/C5 contributes to maintenance of neuropathic pain.

\section{A R T I C L E I N F O}

\section{Article history:}

Received 3 September 2015

Received in revised form

24 September 2015

Accepted 25 September 2015

Available online 30 September 2015

\section{Keywords:}

Amygdala

Aversive place-conditioning

Descending pain control

Neuropathic pain

Transient receptor potential channels 4 and

\begin{abstract}
A B S T R A C T
Pain per se may increase anxiety and conversely, anxiety may increase pain. Therefore, a positive feedback loop between anxiety and pain possibly contributes to pain and suffering in some pathophysiological pain conditions, such as that induced by peripheral nerve injury. Recent results indicate that transient receptor channels 4 and 5 (TRPC4/C5) in the amygdala have anxiogenic effects in rodents, while their role in chronic pain conditions is not known. Here, we studied whether the amygdaloid TRPC4/C5 that are known to have anxiogenic properties contribute to the maintenance of sensory or affective aspects of pain in an experimental model of peripheral neuropathy. Rats with a spared nerve injury (SNI) model of neuropathy in the left hind limb had a chronic cannula for microinjections of drugs into the right amygdala or the internal capsule (a control site). Sensory pain was assessed by determining mechanical hypersensitivity with calibrated monofilaments and affective pain by determining aversive place-conditioning. Amygdaloid treatment with ML-204, a TRPC4/C5 antagonist, produced a dose-related (5-10 $\mu \mathrm{g}$ ) antihypersensitivity effect, without obvious side-effects. Additionally, amygdaloid administration of ML-204 reduced affective-like pain behavior. In the internal capsule, ML-204 had no effect on hypersensitivity or affective-like pain in SNI animals. In healthy controls, amygdaloid administration of ML-204 failed to influence pain behavior induced by mechanical stimulation or noxious heat. The results indicate that the amygdaloid TRPC4/C5 contribute to maintenance of pain hypersensitivity and pain affect in neuropathy.
\end{abstract} (C) 2015 Elsevier Ireland Ltd. All rights reserved.

\section{Introduction}

Amygdala has an important role in primary emotions, such as fear [10]. Amygdala, particularly its lateral capsular subdivision within the central nucleus (CeA), the main output pathway to the brainstem, is also involved in processing and regulation of

\footnotetext{
Abbreviations: ANOVA, analysis of variance; DMSO, dimethylsulfoxide; CeA, central nucleus of the amygdala; i.c., internal capsule; i.t., intrathecal; SNI, spared nerve injury; TRPC4/C5, transient receptor channel 4/5.

* Corresponding author.

E-mail address: antti.pertovaara@helsinki.fi (A. Pertovaara).

1 These authors had an equal contribution to this work.
}

emotional aspects of pain and, through its efferent brainstem projections, in descending control of spinal pain-relay neurons $[14,16]$. Recently, it was demonstrated that among multiple amygdaloid mechanisms involved in the control of anxiety-driven behaviors are transient potential channels 4 and 5 (TRPC4/C5) that are members of the TRPC1/4/5 subfamily and that gate afferent amygdaloid inputs in the lateral nucleus of the amygdala $[17,18]$. The lateral amygdala has direct and indirect projections through the basolateral nucleus to the main amygdaloid output nucleus CeA [10]. It may be hypothesized that TRPC4/C5-expressing amygdaloid neurons through projections to the $\mathrm{CeA}$ may regulate amygdaloid processing of nociception and thereby also amygdaloid outputs to brainstem pain regulation centers. Since, the amygdala is involved 

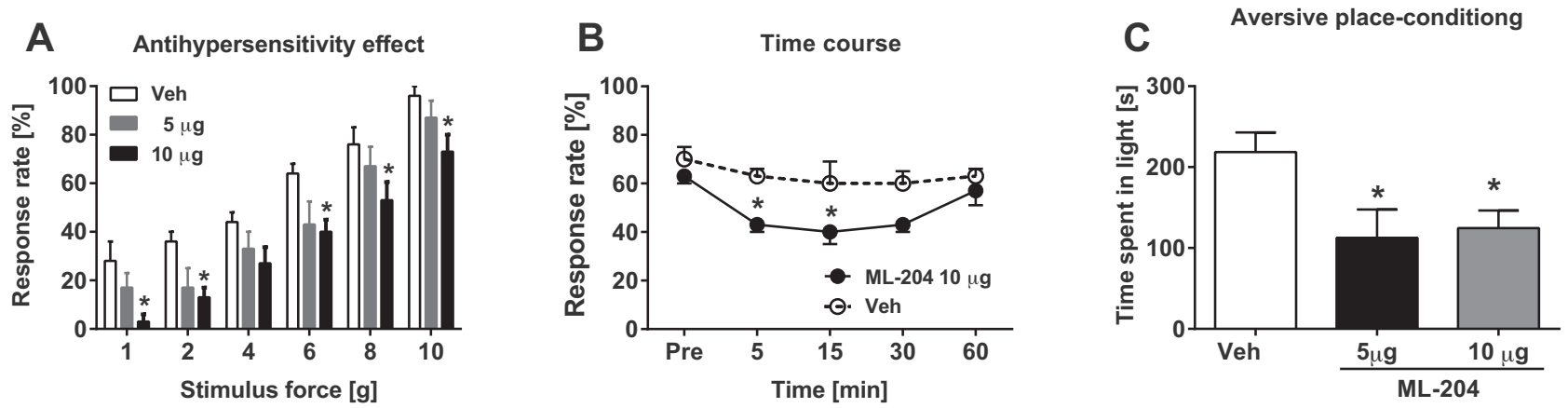

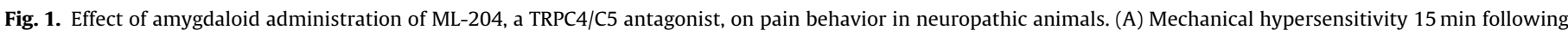

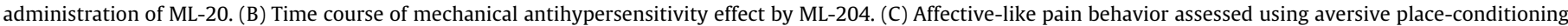
paradigm following administration of ML-204.

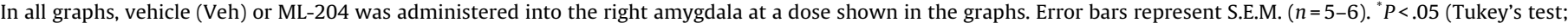
reference: the corresponding Veh-value).

both in pain and affect and has reciprocal connections with the pain system $[14,16]$, it may be speculated that amygdala in general and amygdaloid TRPC4/C5 in particular might be among neural substrates contributing to the comorbidity between affect, such as anxiety, and chronic pain (e.g., see Ref. [2]). To test this hypothesis, we assessed whether microinjection of a TRPC4/C5 antagonist ML204 [12] into the amygdala or a control injection site in the brain influences pain hypersensitivity in animals with an experimental model of chronic neuropathy. The effect of blocking the amygdaloid TRPC4/C5 was separately assessed on the sensory component of neuropathic pain by determining mechanical hypersensitivity and on the affective component of neuropathic pain by determining aversive place-conditioning. For comparison, we determined whether blocking the amygdaloid TRPC4/C5 influences pain behavior in healthy controls.

\section{Materials and methods}

\subsection{Animals}

The experiments were performed with adult male HannoverWistar rats (Harlan, Horst, The Netherlands) weighing 180-280 g. The experimental protocols were approved by the Experimental Animal Ethics Committee of the Provincial Government of Southern Finland (Hämeenlinna, Finland), and the experiments were performed according to the guidelines of European Communities Council Directive of 22 September 2010 (2010/63/EU). All efforts were made to limit distress and to use only the number of animals necessary to produce reliable scientific data. Rats were housed in a 12-h light/dark cycle with food and water access ad libitum.

\subsection{Surgical procedures for producing neuropathy}

For inducing neuropathy, the spared nerve injury (SNI) model, as described by Decosterd and Woolf [5], was adopted. Prior to surgery, the rat was anesthetized with sodium pentobarbital (Orion Pharma, Espoo, Finland), administered intraperitoneally at the dose of $60 \mathrm{mg} / \mathrm{kg}$. Further, doses of pentobarbitone were given at the dose of $15-20 \mathrm{mg} / \mathrm{kg}$ as needed to keep the depth of anesthesia deep enough so that the animal did not react to noxious stimulation. An incision was subsequently made into the skin on the lateral surface of the left thigh, followed by a section through the biceps femoris muscle to expose the sciatic nerve and its terminal branches: the sural, common peroneal and tibial nerves. The common peroneal and tibial nerves were then tightly ligated with 4-0 silk, sectioned distal to the ligation and 3-4 $\mathrm{mm}$ of the distal nerve stump was removed. The sural nerve was left intact. To prevent postopera- tive pain, animals were treated subcutaneously with $0.01 \mathrm{mg} / \mathrm{kg}$ of buprenorphine twice daily for 2-3 days and they were allowed to recover for at least a week before the experiments. Only animals with tactile allodynia-like hypersensitivity (hind limb withdrawal threshold to monofilament stimulation in the operated side $\leq 4 \mathrm{~g}$, which is below the lower 95\% confidence limit of the threshold in unoperated control animals) were selected for this study. SNI model produced mechanical hypersensitivity in all animals of the present study.

\subsection{Cannula insertion and drug injection procedure}

The animals had a guide cannula for drug administration into the right amygdala (contralateral to the peripheral nerve injury) as described in detail earlier [19]. The rationale for choosing the right amygdala was that earlier results have suggested that the right amygdala has a more important role in processing of painrelated signals than the left amygdala [4,6], although not in all conditions [20]. Moreover, another reason for choosing the right amygdala as the treatment target was that in the present study mechanical hypersensitivity was induced in the left hind limb and the amygdala-induced descending control of mechanically evoked pain is expected to be stronger in the contra- than ipsilateral limb $[3,8]$. For placement of the guide cannula (26 gauge; Plastics One, Roanoke, VA, USA), the skull was exposed and a hole drilled for its placement under sodium pentobarbital anesthesia $(60 \mathrm{mg} / \mathrm{kg}$ i.p.). The desired center of injection in the right amygdala was in the capsule lateral of the CeA: $2.1 \mathrm{~mm}$ posterior from the bregma, $4.3 \mathrm{~mm}$ lateral from the midline, and $7.8 \mathrm{~mm}$ ventral from the dura mater [15]. The control injection site was in the right internal capsule: $2.1 \mathrm{~mm}$ posterior from bregma, $3.6 \mathrm{~mm}$ lateral from the midline, and $5.0 \mathrm{~mm}$ ventral from the dura mater. The tip of the guide cannula was positioned $2 \mathrm{~mm}$ above the desired injection site. The cannula was fixed into the skull using a dental screw and dental cement. Drug administration to the brain and experimental protocols started 1 week after fixation of the guide cannula to the skull. A dummy cannula was placed in the guide cannula, except when drug administrations were performed.

\subsection{Drugs and their administration procedure}

ML-204, a selective TRPC4/C5 antagonist [12], and dimethylsulfoxide (DMSO), the vehicle, were purchased from Sigma-Aldrich (St. Louis, MO, USA). ML-204 was dissolved in DMSO (100\%) and administered at the dose of $5 \mu \mathrm{g}$ or $10 \mu \mathrm{g}$. Unilateral infusions of ML-204, or an equivalent volume of vehicle, were made by using 33 gauge injection needles (Plastics One) connected to a $10 \mu \mathrm{l}$ 
A

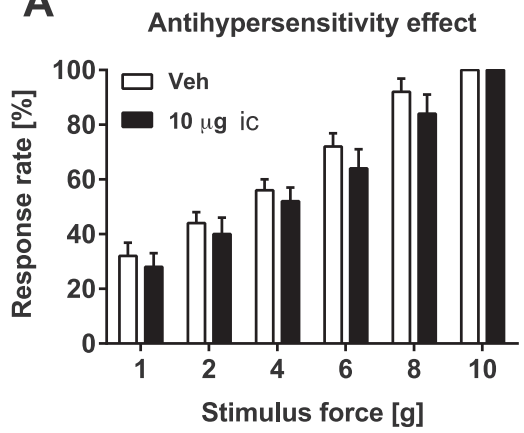

C

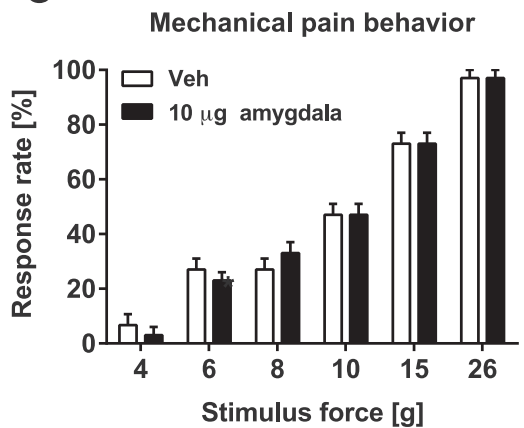

B

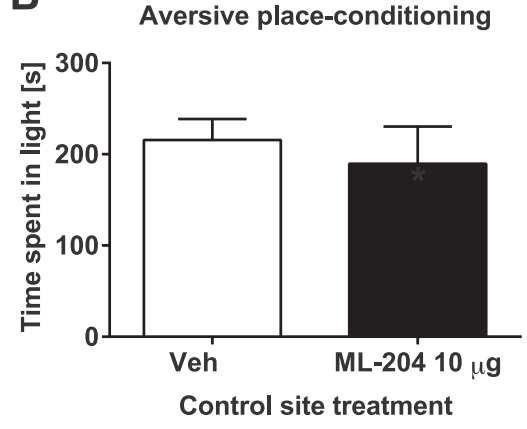

D

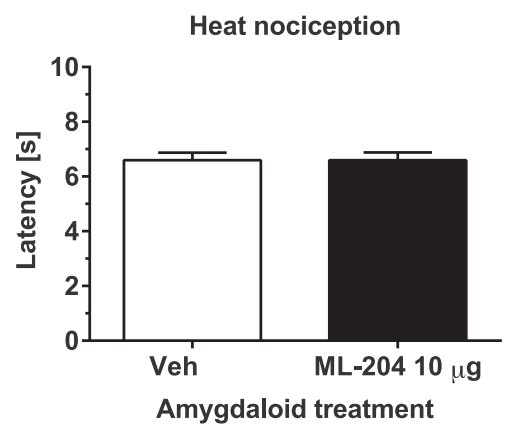

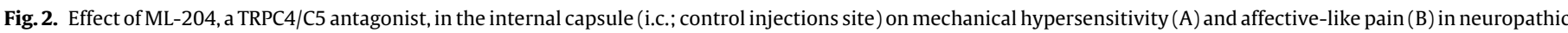
animals. Effect of amygdaloid administration of ML-204 on mechanically evoked pain behavior (C) and heat nociception (D) in healthy controls.

Veh, vehicle. Error bars represent S.E.M. $(n=5-6)$. For other explanations, see the legend for Fig. 1.

Hamilton microsyringe (Hamilton Bonaduz AG, Bonaduz, Switzerland) by polyethylene (PE-10) tubing. The injection needles protruded $2.0 \mathrm{~mm}$ beyond the cannula tips and a $0.5 \mu \mathrm{l}$ volume was injected. The animals were gently restrained during the infusion procedure. The duration of injection was $30 \mathrm{~s}$. Injection needle was retained within the cannula for an additional $20 \mathrm{~s}$ after drug infusion to maximize diffusion and to prevent backflow of the drug into the cannula. At the currently used injection volume, the spread of injection is expected to be close to $1 \mathrm{~mm}$ [13] and thereby cover not only the CeA but also the adjacent amygdaloid subnuclei. Therefore, the currently applied drug injection volume allows concluding whether the drug-induced effect originates in the amygdala rather than pinpointing the effect to one of its subnuclei.

\subsection{Assessment of the sensory-like component of pain behavior}

Before assessment of pain behavior, the rats were habituated to the testing conditions at least in three one-hour sessions during three consecutive days. Mechanically evoked limb withdrawal was considered an index of sensory-like pain and assessed both in neuropathic animals and healthy controls using a calibrated series of monofilaments that in the current experiment produced forces ranging from $1 \mathrm{~g}$ to $10 \mathrm{~g}$ in nerve-injured animals or $4 \mathrm{~g}$ to $26 \mathrm{~g}$ in controls (North Coast Medical, Inc. Morgan Hill, CA, USA). During testing, rats were on a grid, free to move inside a transparent box. The monofilaments were applied below the grid to the foot pad of the left hind limb with increasing force until the rat withdrew its hind limb. At each time point, the paw ipsilateral to the spinal nerve ligation was stimulated five times at each stimulus force with an ascending series of calibrated monofilaments. At each stimulus force, the withdrawal response frequency was determined. When the animal responded by withdrawing the limb to each of the five repetitive monofilament stimulations, the response rate to the studied stimulus force was consider to be $100 \%$. When the animal did not withdraw to any of the five repetitive stimulations, the response rate to the studied stimulus force was considered to be $0 \%$. An increase in the withdrawal response rate was considered to represent mechanical hypersensitivity effect.

In healthy controls, mechanical pain was assessed as described above. Moreover, sensory-like heat pain was assessed in healthy controls by determining limb withdrawal latency induced by noxious heat applied to the plantar skin using radiant heat equipment (Plantar test model 7370, Ugo Basile, Varese, Italy). The cut-off point was set at $15 \mathrm{~s}$.

\subsection{Assessment of the affective-like component of pain behavior}

Place-avoidance test was performed in neuropathic animals, as described earlier [9], to obtain a measure of affective-like pain. Before testing, the animals were habituated to test conditions by spending 1-2 h daily for 2 days in the test box. In the actual testing, the rat was placed within a Plexiglas chamber $(60 \times 30 \times 30 \mathrm{~cm})$ one half of which was painted black on the external surface placed upon an elevated metal grid. The rats were placed over the midline of the chamber and stimulation of the plantar surface of the hind paw initiated with a $26 \mathrm{~g}$ monofilament once every $15 \mathrm{~s}$ for $20 \mathrm{~min}$. When residing within the dark side of the chamber the hind paw of the nerve-injured limb was stimulated. Conversely, the non-operated hind paw was stimulated when residing within the light side of the chamber. Throughout the 20 min test period rats were allowed unrestricted movement throughout the chamber. The percent time spent in the dark side of the chamber during the observation period was determined in each condition for each animal. It is assumed that the more aversive the mechanical stimulation of the hind paw, the more the animal spends time in the light side of the chamber. 


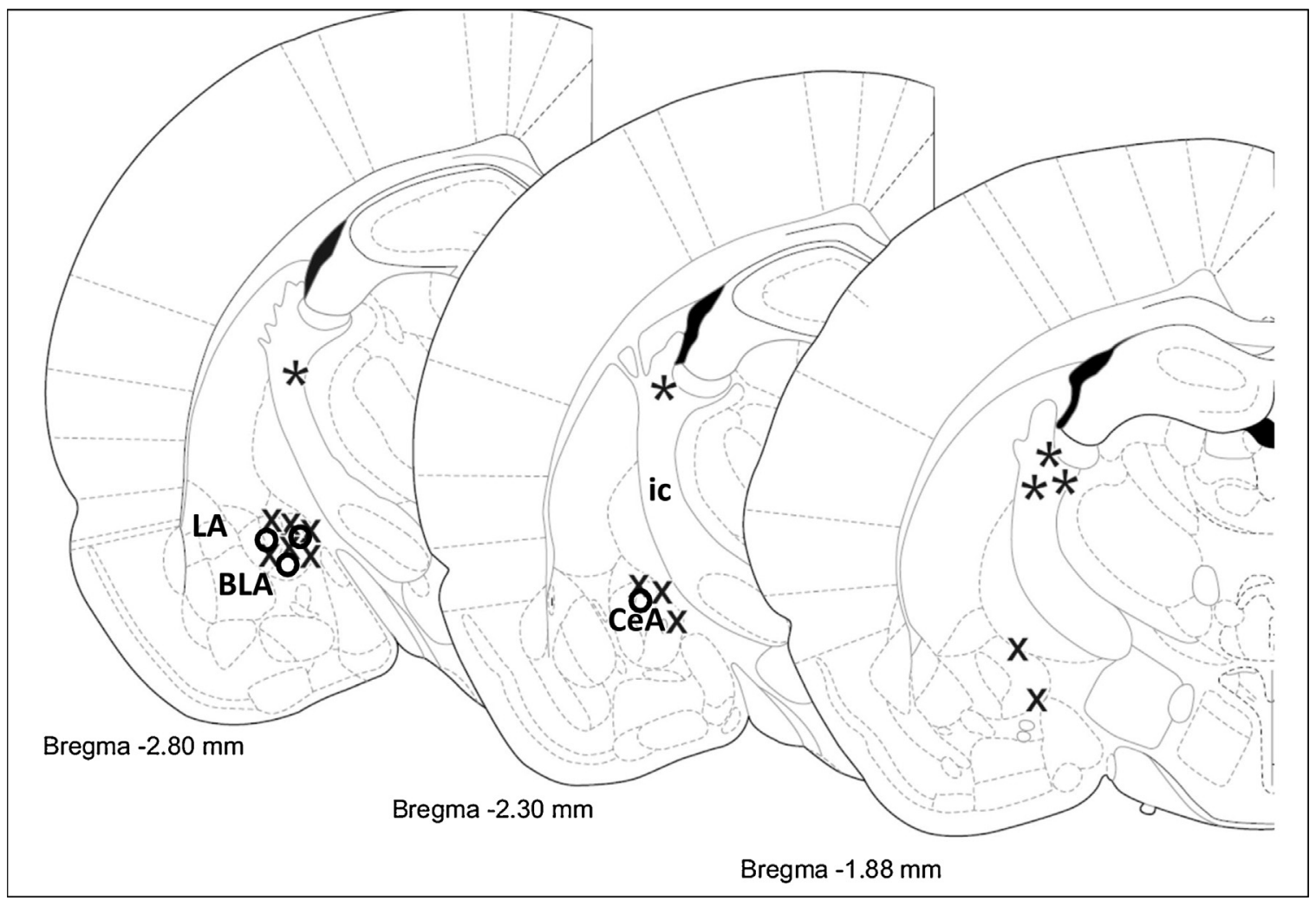

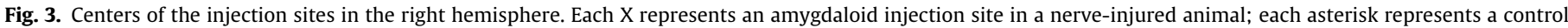

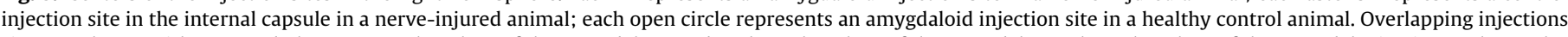

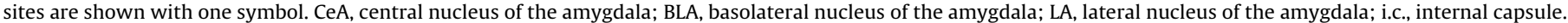

\subsection{Course of study}

There were three separate groups of animals: (i) SNI animals with a cannula in the right amygdala, (ii) SNI animals with a cannula in the right internal capsule, (iii) healthy controls with a cannula in the right amygdala. Experiments were performed at least a week following unilateral installation of the brain cannula into the right amygdala or internal capsule. Mechanically induced limb withdrawal, heat-evoked limb withdrawal and aversive placeconditioning were evaluated on separate days. Pain behavior was tested in the following drug-treatment conditions: vehicle, ML204 at the dose of $5 \mu \mathrm{g}$ or $10 \mu \mathrm{g}$. Limb withdrawal responses were assessed $5,15,30$, and $60 \mathrm{~min}$ after drug administration as well as before it, while place-avoidance was continuously assessed for 20 min after drug administration. The order of testing the drug conditions was varied to avoid serial effects. The interval between testing the different experimental conditions in the same animal was two days.

\subsection{Histology}

At the end of the experiment, rats were sacrificed by an overdose of pentobarbital and the brain was removed and immersed in $4 \%$ formaldehyde. Coronal sections of the brain were cut to verify the site of injection according to the atlas of Paxinos and Watson [15].

\subsection{Statistical analyses}

Data were analyzed using one-way or two-way ANOVA followed by Tukey's test, or by $t$-test. $P<.05$ was considered to represent a significant difference.

\section{Results}

3.1. Effect on pain behavior of neuropathic animals by amygdaloid administration of ML-204

Microinjection of ML-204 (an antagonist of the TRPC4/C5 channels) at doses $0 \mu \mathrm{g}$ (vehicle control), $5 \mu \mathrm{g}$, and $10 \mu \mathrm{g}$ into the right amygdala of neuropathic animals produced a dose-related mechanical antihypersensitivity effect in the injured left $\operatorname{limb}\left(F_{2,84}=18.04\right.$, $P<.0001$; Fig. 1A). The onset of the antihypersensitivity effect occurred within $5 \mathrm{~min}$, the maximum effect was reached at $15 \mathrm{~min}$, and the effect disappeared by $60 \mathrm{~min}$ (Fig. 1B). In the aversive place-conditioning test, ML-204 in the amygdala reduced affectivelike pain behavior as indicated by the reduction in time spent in light $\left(F_{2,14}=4.83, P=.025\right.$; Fig. $\left.1 C\right)$. The suppression of affective-like pain behavior induced by amygdaloid administration of ML-204 at a dose of $5 \mu \mathrm{g}$ was of equal magnitude as at the dose of $10 \mu \mathrm{g}$ (Fig. 1C). No obvious side-effects were observed following amygdaloid administration of ML-204 at doses 5-10 $\mu \mathrm{g}$.

\subsection{Effect on pain behavior of neuropathic animals by ML-204 at a control injection site}

Microinjection of ML-204 (10 $\mu \mathrm{g})$ into the control injection site, the right internal capsule, failed to attenuate mechanical hypersensitivity $\left(F_{1,48}=2.72\right.$; Fig. $\left.2 \mathrm{~A}\right)$. Moreover, ML-204 at the dose of $10 \mu \mathrm{g}$ failed to influence aversive place-conditioning ( $t_{9}=0.59$; Fig. $\left.2 \mathrm{~B}\right)$.

\subsection{Effect of amygdaloid administration of ML-204 on pain behavior in healthy controls}

In healthy controls, administration of ML-204 (10 $\mu \mathrm{g})$ into the right amygdala failed to influence pain behavior induced by 
mechanical stimulation $\left(F_{1,60}=0.02\right.$; Fig. $\left.2 \mathrm{C}\right)$ or noxious heating $\left(t_{10}=0\right.$; Fig. 2D).

\subsection{Centers of brain injection sites}

The centers of the amygdaloid injection sites were in or immediately adjacent to the CeA in the right hemisphere (Fig. 3). The centers of control injection sites were in the right internal capsule (Fig. 3).

\section{Discussion}

The present results indicate that pharmacological blocking of the TRPC4/C5 in the amygdala attenuates sensory- and affective-like pain in neuropathy as indicated by the mechanical antihypersensitivity effect and the decrease of time spent in light in the aversive place-conditioning test, respectively. The currently used microinjection volume of $0.5 \mu \mathrm{l}$ does not allow pinpointing the critical site of drug action at a subnucleus level. However, the finding that the TRPC4/C5 antagonist failed to influence pain behavior of neuropathic animals when microinjected into a control site, the internal capsule, supports the interpretation that the TRPC4/C5 antagonist-induced suppression of neuropathic pain behavior in the present study was due to action on the amygdala rather than elsewhere in the brain. In line with this, it has been shown that TRPC4/C5 in the amygdala are expressed particularly in the lateral nucleus $[17,18]$, which has direct and indirect intra-amygdaloid projections to the CeA [10], the main sensory output nucleus that is involved in processing of pain-related signals and that through its connections to the brainstem can contribute to the descending control of pain $[14,16]$.

Pharmacological blocking of the amygdaloid TRPC4/C5 failed to influence pain behavior in healthy controls. This finding suggests that in chronic painful neuropathy a change in the function of amygdala takes place, after which the amygdaloid TRPC4/C5 have a significant pain facilitatory effect. Chronic neuropathy, including that induced by the currently used experimental animal model, is known to induce anxiety that is associated with ongoing pain and pain hypersensitivity [11]. Moreover, blocking the amygdaloid TRPC4/C5 was shown to reduce anxiety in earlier studies $[17,18,22]$ and suppress neuropathic pain behavior in the present study. Together these findings suggest that the amygdaloid TRPC4/C5 are among potential underlying mechanisms for comorbidity of pain and affect in chronic neuropathy.

While the effects of amygdaloid TRPC4/C5 on neuropathic pain have not been studied earlier, there are earlier studies that have assessed pain behavior in various pain models following systemic pharmacological blocking of TRPC4/C5 or genetic blocking of the TRPC4. These studies have demonstrated that e.g., chemically induced bladder overactivity and visceral pain are reduced by a systemic block of TRPC4/C5 [1,21], which effects may, at least partly be explained by a peripheral action [21]. Only negligible differences were observed in somatically induced pain behavior or in the effect of joint inflammation between control and TRPC4 knockout rats [21]. It is noteworthy that a model of peripheral neuropathy induced by constriction injury of the sciatic or trigeminal nerve failed to show markedly different pain behavior between controls and TRPC4 knockout rats [21], whereas pharmacological block of the amygdaloid TRPC4/C5 in the SNI model of neuropathy attenuated hypersensitivity in the present study. Among potential explanations for this difference are that genetic blocking affecting TRPC4 only and pharmacological blocking influencing amygdaloid TRPC4 and TRPC5 may have different effects on neuropathic pain or that constriction injury and SNI models of neuropathy induce at least partly different pathophysiological changes in the central pain signaling system [7]. Additionally, various differences in experimental conditions, such as the methods for assessing sensory- and affective-like pain or local amygdaloid versus systemic blocking of channels may have contributed to the difference.

\section{Conclusions}

The present results suggest that the amygdaloid TRPC4/C5 that in earlier studies were shown to be involved in control of innate fear $[17,18]$ contribute to regulation of pain hypersensitivity and pain affect in neuropathy. It is hypothesized that the amygdaloid TRPC4/C5 might exert a role in comorbidity between affect and chronic pain and thereby, they might provide therapeutic targets in affective disorder-associated chronic neuropathic pain conditions.

\section{Conflicts of interest}

One of the authors (AK) is an employee of a pharmaceutical company (Orion Corporation, Orion Pharma, Turku, Finland).

\section{Acknowledgements}

The study was financially supported by the Academy of Finland, the 375th Anniversary Grant of the Faculty of Medicine, University of Helsinki, and the Sigrid Jusélius Foundation, Helsinki, Finland. M.A.Y. whose current address is Ankara University Faculty of Medicine, Ankara, Turkey was supported by the European Union Erasmus Program.

\section{References}

[1] M. Boudes, P. Uvin, S. Pinto, M. Freichel, L. Birnbaumer, T. Voets, D. De Ridder R. Vennekens, Crucial role of TRPC1 and TRPC4 in cystitis-induced neuronal sprouting and bladder overactivity, PLoS One 8 (2013) e69550.

[2] N. Bourbia, A. Pertovaara, Is finding the common biological link(s) between pain and affect an infinity quest? Scand. J. Pain 2 (2011) 137-138.

[3] N. Bourbia, B. Sagalajev, A. Pertovaara, Descending effect on spinal nociception by amygdaloid glutamate varies with the submodality of noxious test stimulation, Neurosci. Lett. 570 (2014) 26-31.

[4] Y. Carrasquillo, R.W. Gereau, Hemispheric lateralization of a molecular signal for pain modulation in the amygdala, Mol. Pain 4 (2008) 24

[5] I. Decosterd, C.J. Woolf, Spared nerve injury: an animal model of persistent peripheral neuropathic pain, Pain 87 (2000) 149-158

[6] G. Ji, V. Neugebauer, Hemispheric lateralization of pain processing by amygdala neurons, J. Neurophysiol. 102 (2009) 2253-2264.

[7] T. Kohno, K.A. Moore, H. Baba, C.J. Woolf, Peripheral nerve injury alters excitatory synaptic transmission in lamina II of the rat dorsal horn, J. Physiol. 548 (2003) 131-138.

[8] B.J. Kolber, M.C. Montana, Y. Carrasquillo, J. Xu, S.F. Heinemann, L.J. Muglia, R.W. Gereau IV, Activation of metabotropic glutamate receptor 5 in the amygdala modulates pain-like behavior, J. Neurosci. 30 (2010) 8203-8213.

[9] C.J. LaBuda, P.N. Fuchs, A behavioral test paradigm to measure the aversive quality of inflammatory and neuropathic pain in rats, Exp. Neurol. 163 (2000) 490-494.

[10] J. LeDoux, The amygdala, Curr. Biol. 17 (2007) R868-R874.

[11] H. Leite-Almeida, J.J. Cerqueira, H. Wei, N. Ribeiro-Costa, H. Anjos-Martins, N. Sousa, A. Pertovaara, A. Almeida, Differential effects of left/right neuropathy on rats' anxiety and cognitive behavior, Pain 153 (2012) 2218-2225.

[12] M. Miller, J. Shi, Y. Zhu, M. Kustov, J.B. Tian, A. Stevens, M. Wu, J. Xu, S. Long, P. Yang, A.V. Zholos, J.M. Salovich, C.D. Weaver, C.R. Hopkins, C.W. Lindsley, O. McManus, M. Li, M.X. Zhu, Identification of ML204, a novel potent antagonist that selectively modulates native TRPC4/C5 ion channels, J. Biol. Chem. 286 (2011) 33436-33446.

[13] R.D. Myers, Injection of solutions into cerebral tissue: relation between volume and diffusion, Physiol. Behav. 1 (1966) 171-174.

[14] V. Neugebauer, V. Galhardo, S. Maione, S. Mackey, Forebrain pain mechanisms, Brain Res. Rev. 60 (2009) 226-242.

[15] G. Paxinos, C. Watson, The Rat Brain in Stereotaxic Coordinates, sixth ed., Academic Press, New York, 2008.

[16] A. Pertovaara, A. Almeida, Descending inhibitory systems, Handb. Clin. Neurol. 81 (2006) 179-192.

[17] A. Riccio, Y. Li, J. Moon, K.S. Kim, K.S. Smith, U. Rudolph, S. Gapon, G.L. Yao, E Tsvetkov, S.J. Rodig, A. Van't Veer, E.G. Meloni, W.A. Carlezon Jr., V.Y Bolshakov, D.E. Clapham, Essential role for TRPC5 in amygdala function and fear-related behavior, Cell 137 (2009) 761-772 
[18] A. Riccio, Y. Li, E. Tsvetkov, S. Gapon, G.L. Yao, K.S. Smith, E. Engin, U. Rudolph, V.Y. Bolshakov, D.E. Clapham, Decreased anxiety-like behavior and Goq/11-dependent responses in the amygdala of mice lacking TRPC4 channels, J. Neurosci. 34 (2014) 3653-3667.

[19] B. Sagalajev, N. Bourbia, E. Beloushko, H. Wei, A. Pertovaara, Bidirectional amygdaloid control of neuropathic hypersensitivity mediated by descending serotonergic pathways acting on spinal $5-\mathrm{HT}_{3}$ and 5- $\mathrm{HT}_{1 \mathrm{~A}}$ receptors, Behav. Brain Res. 282 (2015) 14-24.

[20] C.A. Spuz, G.S. Borszcz, NMDA or non-NMDA receptor antagonism within the amygdaloid central nucleus suppresses the affective dimension of pain in rats: evidence for hemispheric synergy, J. Pain 13 (2012) 328-337.
[21] K.N. Westlund, L.P. Zhang, F. Ma, R. Nesemeier, J.C. Ruiz, E.M. Ostertag J.C. Crawford, K. Babinski, M.M. Marcinkiewicz, A rat knockout model implicates TRPC4 in visceral pain sensation, Neuroscience 262 (2014) 165-175.

[22] L.P. Yang, F.J. Jiang, G.S. Wu, K. Deng, M. Wen, X. Zhou, X. Hong, M.X. Zhu, H.R. Luo, Acute treatment with a novel TRPC4/C5 channel inhibitor produces antidepressant and anxiolytic-like effects in mice, PLoS One 10 (2015) e0136255. 